\title{
A SIMULAÇÃO NO ENSINO DE ENFERMAGEM: UMA ANÁLISE CONCEITUAL
}

\section{SIMULATION IN NURSING TEACHING: A CONCEPTUAL ANALYSIS}

\section{SIMULACIÓN EN EDUCACIÓN DE ENFERMERÍA: UN ANÁLISIS CONCEPTUAL}

Raphael Raniere de Oliveira Costa ${ }^{1}$, Soraya Maria de Medeiros ${ }^{2}$, José Carlos Amado Martins ${ }^{3}$, Bertha Cruz Enders ${ }^{4}$, Ana Luisa Brandão de Carvalho Lira ${ }^{5}$, Marília Souto de Araújo ${ }^{6}$

\section{RESUMO}

Objetivo: analisar o conceito simulação no ensino de enfermagem. Método: trata-se de uma análise de conceito, seguindo o método de Walker e Avant, na qual foi realizada uma revisão integrativa da literatura nas bases de dados Scientific Electronic Library Online, Base de Dados de Enfermagem e Literatura Latino-americana e do Caribe em Ciências da Saúde (LILACS). Amostra final foi de 17 estudos. Resultados: 0 conceito elaborado para simulação no ensino de enfermagem foi: uma estratégia de ensino que utiliza-se de tecnologias, replicando cenários simulando a prática, em ambiente controlado e realista, onde o estudante participa ativamente do processo de ensino e aprendizagem, almejando praticar exaustivamente, aprender, refletir e avaliar produtos e processos. Conclusão: os achados auxiliam na construção de instrumentos de pesquisas e a compreensão na área de investigação do fenômeno estudado.

Descritores: Simulação; Ensino de enfermagem; Formação de conceito.

\section{ABSTRACT}

Objective: to analyze the concept of simulation in nursing teaching. Method: this is a concept analysis, following the Walker and Avant method, in which an integrative review of the literature was carried out in the databases Scientific Electronic Library Online, Database of Nursing and Latin American and Caribbean Literature in Health Sciences (LILACS). Final sample was from 17 studies. Results: The concept developed for simulation in nursing teaching was: a teaching strategy that uses technologies replicating scenarios simulating the practice, in a controlled and realistic environment, where the student participates actively in the teaching and learning process, aiming to practice comprehensively, to learn, reflect and evaluate products and processes. Conclusion: the findings help in the construction of research instruments and the understanding in the research area of the phenomenon studied. Descriptors: Simulation; Education, nursing; Concept formation.

\section{RESUMEN}

Objetivo: analizar el concepto simulación en la educación de enfermería. Método: se trata de un análisis de concepto, siguiendo el método de Walker y Avant, en la que se llevó a cabo una revisión integradora de la literatura en las bases de datos Scientific Electronic Library, Base de Datos de Enfermería y Literatura Latinoamericana y de Caribe en Ciencias de la Salud (LILACS). La muestra final fue de 17 estudios. Resultados: el concepto desarrollado para la simulación en la educación de enfermería fue: una estrategia de enseñanza que utiliza la tecnología replicando escenarios con simulación de la práctica, en un entorno controlado, realista, donde el estudiante participa activamente del proceso de enseñanza y aprendizaje, con el objetivo de practicar ampliamente, el aprendizaje, reflexionar y evaluar los productos y procesos. Conclusión: los resultados ayudan a construir instrumentos de investigación y conocimiento en el campo de la investigación del fenómeno estudiado.

Descriptores: Simulación; Educación en enfermería; Formación de concepto.

${ }^{1}$ Graduado em Enfermagem. Doutor em Enfermagem pela Universidade Federal do Rio Grande do Norte. Docente da Escola Multicampi de Ciências Médicas do Rio Grande do Norte - UFRN. ${ }^{2}$ Graduada em Enfermagem. Doutora em Enfermagem pela Universidade de São Paulo de Ribeirão Preto. Docente do Departamento de Enfermagem da Universidade Federal do Rio Grande do Norte - UFRN. ${ }^{3}$ Graduado em Enfermagem. Doutor em Ciências de Enfermagem pela Universidade do Porto/Portugal. Professor Coordenador na Escola Superior de Enfermagem de Coimbra /Portugal - ESEnfC. ${ }^{4}$ Graduada em Enfermagem. Doutora em Enfermagem pela Texas Womans University/Dallas/USA. Professora Titular do Departamento de Enfermagem da Universidade Federal do Rio Grande do Norte - UFRN. ${ }^{5}$ Graduada em Enfermagem. Doutora em Enfermagem. Docente do Departamento de Enfermagem da Universidade Federal do Rio Grande do Norte - UFRN. ${ }^{6}$ Graduada em Enfermagem. Mestranda do Programa de Pós-Graduação em Enfermagem da Universidade Federal do Rio Grande do Norte - UFRN.

Como citar este artigo:

Costa RRO, Medeiros SM, Martins JCA, et al. A Simulação no ensino de enfermagem: uma análise conceitual. Revista de Enfermagem do Centro-Oeste Mineiro. 2018;8:e1928. [Access__]; Available in:___ DOI: http://dx.doi.org/10.19175/recom.v7i0.1928 


\section{INTRODUÇÃO}

Embora incipiente em muitos países, a simulação é uma estratégia em expansão no contexto da formação de futuros profissionais da enfermagem. Seu uso perpassa a ideia de uma formação mais complexa e que atenda às necessidades do mercado de trabalho atual, cada vez mais exigente ${ }^{(1)}$. Entre as exigências do mercado de trabalho em enfermagem, cita-se a qualidade do cuidado ${ }^{(2)}$.

No ensino de Enfermagem, a simulação é uma estratégia que vem ganhando adeptos. A partir do ano de 2010, nota-se uma expansão nos estudos referente a essa estratégia. Países como Estados Unidos, Austrália e Reino Unido têm se destacado na produção do conhecimento sobre esse fenômeno ${ }^{(1)}$.

É válido considerar que essa estratégia tem sido utilizada em diversos contextos do ensino de Enfermagem. Uma revisão da literatura apontou seu uso na graduação, pós-graduação e também na educação permanente e continuada ${ }^{(1)}$. No Brasil, é possível identificar o seu uso também no nível médio, na formação de técnicos de enfermagem ${ }^{(3)}$.

A simulação é uma estratégia utilizada em diversas áreas do conhecimento. Seu uso tem sido divulgado em áreas distintas como na aviação (os pioneiros da simulação realística), no direito e em diversas disciplinas da área da saúde e na Enfermagem. Ao considerar a vasta utilização e aplicabilidade da simulação no ensino de Enfermagem, percebe-se uma variedade de conceitos utilizados para caracterizar essa estratégia. Simulação, simulação clínica, simulação realística e simulação em saúde são algumas nomenclaturas que têm sido referenciadas na literatura.

Por ser uma estratégia proveniente de outras áreas do conhecimento e, ao considerar o caráter recente de sua aplicação no ensino de enfermagem, é válido compreender a utilização do conceito de simulação no contexto do ensino de Enfermagem.

No contexto da produção do conhecimento de Enfermagem, ao clarear um conceito que ainda é vago, pode-se contribuir para a construção de afirmações ou hipóteses que permitam uma reflexão precisa sobre a relação entre conceitos e construção e análise de teorias. Além disso, a construção de conhecimento próprio é de fundamental relevância para a manutenção e reafirmação da Enfermagem enquanto ciência ${ }^{(4)}$.

Dessa forma, identificar os elementos que compõe o conceito em análise é um passo importante nesse processo de construção do conhecimento. Para o levantamento dos dados, construiu-se a seguinte questão norteadora: como a literatura caracteriza o conceito simulação aplicada ao ensino de enfermagem? Assim, o objetivo deste estudo foi analisar o conceito simulação no ensino de enfermagem.

\section{MÉTODOS}

Trata-se de um estudo conceitual a partir do método de análise proposto por Walker e Avant $^{(4)}$. As autoras apontam oito passos para a análise conceitual, a saber:

1 - Seleção do conceito: a seleção do conceito é uma etapa que deve ser feita com cuidado. É importante escolher conceitos que são pouco explorados em algumas áreas do conhecimento e também refletir no interesse do pesquisador $^{(4)}$.

2 - Determinação dos objetivos da análise: é uma etapa que deve ser decidida pelo pesquisador. Existem diferentes objetivos que podem ser contemplados a partir da análise como, por exemplo, esclarecer o significado de um conceito existente, desenvolver uma definição operacional, desenvolver um instrumento de pesquisa, entre outros ${ }^{(4)}$.

3 - Identificação dos possíveis usos do conceito: Usando diversas fontes de busca e conhecimento, esta etapa objetiva compreender a natureza do conceito. Essa identificação não se limita às literaturas específicas (como enfermagem ou médica). Além disso, essa revisão da literatura ajuda a compreender ou validar as escolhas finais dos atributos e fornece evidências para a análise $\mathrm{e}^{(4)}$.

4 - Identificação dos atributos: determinar os atributos de definição de um conceito é uma etapa fundamental da análise de conceito. Nessa etapa, o esforço é para mostrar o conjunto de atributos que são mais frequentemente associados ao conceito ${ }^{(4)}$.

5 - Identificação de um caso modelo: um caso modelo é uma forma de exemplificar a utilização do conceito. Este caso demostra todos os atributos de definição do conceito, podem ser reais, encontrados na literatura ou criados pelo autor ${ }^{(4)}$. 
6 - Identificação dos casos adicionais: os casos adicionais são casos que não são exatamente os mesmos que o conceito ${ }^{(4)}$.

7 - Identificação dos antecedentes e consequentes: os antecedentes são os eventos que devem ocorrer antes da ocorrência do conceito. Já os consequentes são os resultados do conceito $^{(4)}$.

Para a construção dessa análise, foi realizada uma revisão integrativa da literatura. Para a revisão, foram utilizadas as seguintes etapas: identificação da questão de pesquisa e objetivo do estudo, busca da literatura, avaliação dos dados, análise dos dados e apresentação ${ }^{(5,6)}$.

Para a seleção dos artigos foi realizada uma busca nas bases de dados Scientific Electronic Library Online (SciELO), Base de Dados de Enfermagem (BDENF) e Literatura Latinoamericana e do Caribe em Ciências da Saúde (LILACS), utilizando-se os Descritores em Ciências da Saúde (DeCS) e do Medical Subject Headings (MeSH), respectivamente: simulação, ensino, educação em enfermagem (simulation, teaching, $e$ nursing education). A busca dos estudos publicados no período de 2010 a 2015 foi realizada no mês de janeiro de 2016.

Foram utilizados os seguintes critérios de inclusão: artigos em português, espanhol ou inglês; textos completos, livres, disponíveis e gratuitos, que abordassem a simulação no ensino de enfermagem (graduação, nível médio e/ou pós-graduação). Foram excluídos os artigos com título ou resumo que não associavam a palavra "simulação" ao ensino de enfermagem - por se tratar de uma análise do conceito no contexto da enfermagem - os editoriais, as teses e dissertações.

Para a identificação dos atributos, antecedentes e consequentes, foram criadas algumas questões norteadoras, a saber: atributos: Como está definido o conceito de simulação no ensino de enfermagem? Quais são as particularidades que o conceito em análise apresenta? Antecedentes: Que acontecimentos contribuem para a proximidade e para a existência do conceito de simulação no ensino de enfermagem? Consequentes: $O$ que resultou após a aplicação do conceito de simulação no ensino de enfermagem?

Para a análise do material foi realizada uma busca e leitura minuciosas do material analisado. A Figura 1 apresenta síntese do processo de busca e o número de artigos selecionados de acordo com cada base de dados pesquisada.

Figura 1 - Processo de busca dos estudos e seleção final. Natal, 2016.

\begin{tabular}{|c|c|c|c|c|c|}
\hline $\begin{array}{l}\text { Base de } \\
\text { dados }\end{array}$ & Cruzamento & $\begin{array}{l}\text { Número de artigos } \\
\text { a partir do } \\
\text { cruzamento }\end{array}$ & $\begin{array}{c}\text { Aplicação dos critérios } \\
\text { de inclusão }\end{array}$ & $\begin{array}{c}\text { Amostra } \\
\text { parcial }\end{array}$ & $\begin{array}{c}\text { Amostra } \\
\text { final }\end{array}$ \\
\hline & $\begin{array}{l}\text { (simulation) AND (teaching) AND } \\
\text { (education, nursing) }\end{array}$ & 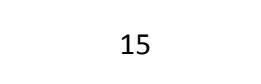 & 5 & \multirow{2}{*}{6} & \multirow{4}{*}{17} \\
\hline SCIELO & $\begin{array}{l}\text { (simulação AND (ensino) AND } \\
\text { (educação em enfermagem) }\end{array}$ & 5 & 1 & & \\
\hline LILACS & $\begin{array}{l}\text { (simulação) AND (ensino) AND } \\
\text { (educação em enfermagem) }\end{array}$ & 27 & 10 & 10 & \\
\hline BDENF & $\begin{array}{l}\text { (simulação) AND (ensino) AND } \\
\text { (educação em enfermagem) }\end{array}$ & 19 & 1 & 1 & \\
\hline
\end{tabular}

Fonte: Autoria própria (2016).

Após a busca dos manuscritos, os dados foram organizados em planilhas no Excel. As planilhas foram organizadas a partir da codificação dos artigos incluídos, atributos, antecedentes e consequentes e analisados os conteúdos a partir da análise de conteúdo de Bardin ${ }^{(7)}$.

\section{RESULTADOS E DISCUSSÃO}

Os estudos analisados foram publicados entre 2010 e 2015, sendo a maior frequência no ano de 2011 (29\%) e em 2015, (29\%), correspondendo a $58 \%$ da amostra. No que se refere à origem de publicação, a maioria dos manuscritos são de origem brasileira (76\%), seguido de Portugal (12\%), México (6\%) e Estados Unidos da América (6\%).

Além disso, a maioria dos estudos relatava o uso da simulação em nível de graduação em enfermagem (82\%). Apenas $12 \%$ dos manuscritos referenciaram o uso dessa ferramenta no nível médio e $6 \%$ na pós-graduação em enfermagem.

\section{Identificação dos possíveis usos do conceito}

A revisão da literatura permitiu identificar que o conceito de simulação no ensino de enfermagem é utilizado em diversas áreas do 
conhecimento dessa disciplina. Ressalta-se a variedade de compreensão e definição. Entretanto, outros estudos incluídos na revisão da literatura não deixaram claro o conceito ou definição empregada. Na Figura 2, são apresentadas algumas definições do conceito analisado.

Figura 2 - Apresentação de definições do conceito de simulação no ensino de enfermagem presentes na literatura. Natal, 2016.

\begin{tabular}{|c|c|}
\hline Referência & Definição \\
\hline $\begin{array}{l}\text { Santos MC, Leite MCL, Heck RM. A possibilidade de } \\
\text { contribuição da acupuntura no ensino da simulação } \\
\text { clínica em enfermagem. = Rev. Gaúcha Enferm. } \\
\text { (Online). 2011; 32(1):185-188. }\end{array}$ & $\begin{array}{l}\text { "espaço protegido que simula cenários da prática de cuidados à saúde } \\
\text { para pequenos grupos de acadêmicos de Enfermagem, onde os } \\
\text { estudantes realizam atendimentos em pacientes simulados, realizam } \\
\text { procedimentos em manequins e/ou bonecos, entre outras atividades } \\
\text { pedagógicas problematizadoras e clínicas" }\end{array}$ \\
\hline $\begin{array}{l}\text { Santos MC, Leite MCL.A avaliação das aprendizagens } \\
\text { na prática da simulação em Enfermagem como } \\
\text { feedback de ensino.Rev. Gaúcha Enferm. (Online). } \\
\text { 2010; } 31(3): 552-556 .\end{array}$ & $\begin{array}{l}\text { "espaços protegidos que simulam cenários da prática de cuidados à } \\
\text { saúde. Os estudantes realizam atendimentos em pacientes simulados, } \\
\text { realizam procedimentos em manequins e/ou bonecos e são } \\
\text { acompanhados por um professor-facilitador que avalia o desempenho } \\
\text { das capacidades voltadas ao perfil do profissional a ser formado" }\end{array}$ \\
\hline $\begin{array}{l}\text { Smithburger PL, et al. Advancing interprofessional } \\
\text { education through the use of high fidelity human } \\
\text { patient simulators. Pharmacy Practice (Internet). } \\
\text { 2013; } 11(2): 61-65 \text {. }\end{array}$ & "Atividade ou evento que replica a prática" \\
\hline $\begin{array}{l}\text { Costa RROC, et al. O uso da simulação no contexto da } \\
\text { educação e formação em saúde e enfermagem: uma } \\
\text { reflexão acadêmica. Revista Espaço para a Saúde. } \\
\text { 2015; 16(1): 59-65. }\end{array}$ & $\begin{array}{l}\text { "uma metodologia que reproduz situações reais permitindo ao aluno } \\
\text { um papel ativo na aquisição dos conceitos necessários para a } \\
\text { compreensão e resolução do problema, enquanto que o professor } \\
\text { adota uma postura de condutor ou facilitador" }\end{array}$ \\
\hline $\begin{array}{l}\text { Salvador PT, et al. Tecnologia no ensino de } \\
\text { enfermagem. Revista Baiana de Enfermagem } \\
\text { Salvador. 2015; 29(1): 33-41. }\end{array}$ & $\begin{array}{l}\text { "uma ferramenta tecnológica ímpar para a qualificação do ensino do } \\
\text { enfermeiro, sobretudo nos aspectos referentes às situações clínicas, } \\
\text { que tanto envolvem riscos aos seres humanos enquanto não possuem } \\
\text { uma certeza de vivência pelos alunos" }\end{array}$ \\
\hline $\begin{array}{l}\text { Barreto DG, et al. Simulação realística como } \\
\text { estratégia de ensino para o curso de graduação em } \\
\text { enfermagem: revisão integrativa. Revista Baiana de } \\
\text { Enfermagem, Salvador. } 2014 ; 28(2): 208-214 \text {. }\end{array}$ & $\begin{array}{l}\text { "técnica ou uma tecnologia e centra-se na recriação de situações da } \\
\text { vida real. Visa permitir aos estudantes o desempenho prático ou a } \\
\text { aquisição de habilidades em um ambiente seguro" }\end{array}$ \\
\hline $\begin{array}{l}\text { Silveira RCP, Robazzi MLCC. Modelos e inovações em } \\
\text { laboratórios de ensino em enfermagem. R. Enferm. } \\
\text { Cent. O. Min. 2011; } 1(4): 592-602 .\end{array}$ & $\begin{array}{l}\text { "técnica em que se utiliza um simulador, em que o simulador é um } \\
\text { objeto ou representação parcial ou total de uma tarefa a ser replicada" }\end{array}$ \\
\hline $\begin{array}{l}\text { Sanino GEC. O uso da simulação em enfermagem no } \\
\text { Curso Técnico de Enfermagem. J. Health Inform. } 2012 \\
\text { Dezembro; } 4 \text { (Número Especial - SIIENF 2012): 148-51. }\end{array}$ & $\begin{array}{l}\text { "técnica, e não tecnologia, para substituir ou ampliar experiências reais } \\
\text { com experiências guiadas, que evocam ou replicam aspectos } \\
\text { substanciais do mundo real de uma forma totalmente interativa" }\end{array}$ \\
\hline
\end{tabular}
Fonte: Autoria própria (2017).

A simulação no ensino de enfermagem é utilizada em distintas áreas do conhecimento e em diferentes níveis de formação. Entre as áreas de formação, destacam-se a enfermagem fundamental ${ }^{(8-9)}$, enfermagem clínica ${ }^{(2-10-11-12-13-14)}$, ensino de técnicas e procedimentos de enfermagem $^{(2-8-11-15-16-17)}$ e primeiros socorros ${ }^{(3)}$.

Os conceitos expostos refletem algumas convergências e divergências. As convergências circundam a concepção de simulação enquanto disparador de aprendizagem que utiliza de um espaço que simula cenário da prática ${ }^{(9-10-11-13-18-19)}$. Além disso, esses espaços permitem erros sem arriscar a saúde do paciente, aumentando a segurança e o aprimoramento de suas habilidades clínicas ${ }^{(11-15-20)}$.

As divergências apontam para um desacordo entre as concepções de técnica ${ }^{(3,9,20)}$, tecnologia ${ }^{(20)}$ metodologia ${ }^{(8)}$, estratégia ${ }^{(14)}$, atividade $^{(16)}$ e ferramenta ${ }^{(1,3,18,21)}$. A partir dessa identificação, passou-se à extração do conjunto de atributos que são mais frequentemente associados ao conceito.

\section{Atributos}

Os atributos identificados foram: espaço que simula a prática; realismo; uso de manequins; feedback; repetição; técnica ou tecnologia; ambiente controlado; participação ativa do discente; praticar; aprender; avaliar; ferramenta tecnológica ${ }^{(2,3,8,9,10-19,21,22)}$.

O laboratório de enfermagem é um espaço comumente utilizado para a realização de simulações $^{(9)}$. Simuladores de baixa tecnologia, pacientes-padrão, simuladores baseados em computador de mesa, simuladores de tarefas 
complexas e simuladores de paciente são alguns dos recursos que podem ser utilizados em simulações ${ }^{(23)}$. 0 realismo relaciona-se, diretamente, às escolhas das ferramentas, manequins e do cenário construído para a simulação ${ }^{(24)}$.

Enquanto técnica, a simulação divide-se em diferentes etapas: sessão informativa, introdução ao ambiente, reunião informativa sobre o simulador, entrada da teoria, reunião informativa sobre o cenário, sessão de simulação e debriefing ${ }^{(24)}$. Nessas etapas, o estudante é inserido em cenários que, possivelmente, podem encontrar na prática real, é familiarizado com os recursos disponibilizados, pratica e resolve situações problemas e refletem sobre o seu desempenho.

O controle do ambiente permite ao estudante treinar e adquirir habilidades considerando os erros sem que, com isso, seja comprometida a segurança do paciente ${ }^{(3)}$. Nesse processo de ensino e aprendizagem, considera-se a utilização das metodologias ativas de ensino, onde o aluno passa a ser protagonista do aprender $^{(25)}$.

O debriefing, momento da reflexão pósexperiência, é uma etapa em que todos os estudantes podem discutir sobre a cena vivenciada. Nesse momento, os estudantes têm a oportunidade de explorar os cenários vivenciados, ajudando-os a consolidar as informações adquiridas, identificar e refletir sobre as áreas em que podem melhorar ${ }^{(26)}$.

\section{Identificação dos casos adicionais Caso modelo}

Após a exposição dialogada sobre imunização de adultos, um grupo de estudantes está reunido em um espaço que replica a sala de vacina de uma unidade de saúde.

O espaço dispõe de geladeira com réplicas de imunobiológicos, caixas térmicas, luvas, seringas, livros de registros, cartão de vacina e outros materiais que são comumente encontrados em espaços para vacinação de adultos.

Os estudantes treinam exaustivamente o aprazamento do cartão de vacina, a partir de casos hipotéticos; aplicam doses em simuladores de baixa tecnologia e contam com um tutor para tirar eventuais dúvidas durante esse processo.

Após aprender as técnicas e procedimentos, os estudantes são convidados a resolver um caso proposto pelo tutor. $\mathrm{Na}$ situação, os estudantes resolvem o caso, refletem sobre sua atuação no cenário e avaliam o momento, a sua desenvoltura na consolidação do conteúdo estudado e a experiência que levará para a prática real.

\section{Caso contrário}

Após a exposição dialogada sobre imunização de adultos, um grupo de estudantes é orientado para revisar o conteúdo fornecido pelo tutor. Após revisar o conteúdo que foi ministrado em sala de aula, os estudantes são convidados a ir ao estágio a realizar aplicações de doses de imunobiológicos na unidade de saúde e consolidam o conteúdo estudado.

\section{Antecedentes e consequentes do conceito de simulação}

Ao identificar os usos do conceito de simulação aplicado ao ensino de enfermagem, extraíram-se os antecedentes e consequentes do conceito analisado. A Figura 3 sintetiza os achados a partir da revisão da literatura.

Figura 3 - Antecedentes e consequentes do conceito de simulação no ensino de enfermagem identificados na literatura. Natal, 2016.

\begin{tabular}{|l|l|}
\hline \multicolumn{2}{|c|}{ Simulação no ensino de enfermagem } \\
\hline Antecedentes & $\begin{array}{l}\text { Formação diferenciada; segurança do paciente; evidências científicas; lacunas na formação; preceitos } \\
\text { éticos e legais; planejamento; estratégias inovadoras; metodologias ativas; ensino de enfermagem; } \\
\text { aprendizagem significativa; laboratório de enfermagem; aprendizagem experiencial. }\end{array}$ \\
\hline Consequentes & $\begin{array}{l}\text { Desenvolvimento de competências e habilidades; pensamento crítico e reflexivo; autoconfiança; } \\
\text { experiências clínicas; autoeficácia; minimização de erros; formação de excelência; motivação e } \\
\text { autoestima; interdisciplinaridade; melhora o desempenho do aluno; diálogo teórico-prático; resolução de } \\
\text { problemas; autonomia; independência; tomada de decisão; avaliação afetiva, cognitiva e psicomotora; } \\
\text { treino de habilidades; satisfação discente; aprendizagem ativa; diminuição do medo. }\end{array}$ \\
\hline
\end{tabular}

Fonte: Autoria própria (2017).

No contexto atual, a simulação, enquanto estratégia pedagógica, tem sido referenciada no desenvolvimento de competências e habilidades em estudantes de enfermagem ${ }^{(27)}$. Nessa 
perspectiva, diversos estudos têm mostrado a contribuição e abrangência da simulação no ensino de enfermagem.

No que se refere à possibilidade de replicar cenários que reproduzem a prática, esta permite aos estudantes de enfermagem ampliar as suas possibilidades de vivenciar os seus futuros contextos de atuação. Essas experiências podem ser importantes e favorecer uma aprendizagem significativa ${ }^{(18)}$.

O realismo dos cenários reproduzidos dependerá dos recursos disponíveis nas escolas de enfermagem. Além das questões estruturais, é importante ter uma equipe técnica qualificada e docentes com conhecimento sobre a técnica e as etapas da simulação ${ }^{(24)}$.

Para a composição de cenários, a simulação poderá ser acrescida de simuladores de baixa tecnologia, pacientes-padrão, simuladores baseados em computador de mesa, simuladores de tarefas complexas e simuladores de paciente são alguns dos recursos que podem ser utilizados em simulações ${ }^{(23)}$. A depender do objetivo delineado, os docentes e a equipe responsável pela formatação e composição desses cenários selecionam e disponibilizam os equipamentos necessários para a execução da simulação.

É importante destacar que uma das mudanças no ensino da enfermagem é a ênfase no desenvolvimento do espírito crítico, a capacidade de analisar, de avaliar, de questionar, de investigar, de divergir, de argumentar e experimentar ${ }^{(27)}$. Essas mudanças objetivam atender às modificações do mundo atual do trabalho e as exigências do setor saúde ${ }^{(17)}$. Além disso, questões como: a segurança do paciente, as novas exigências de formação dos profissionais da saúde, questões éticas e legais e a diversidade de tecnologias colaboram com o desenvolvimento das capacidades anteriormente referenciadas ${ }^{(2)}$.

No que se refere aos resultados da utilização da simulação no ensino de enfermagem, a literatura tem evidenciado contribuições significativas no processo de ensino e aprendizagem. Uma pesquisa realizada na Universidade de Pittsburgh, nos Estados Unidos, constatou que os estudantes que frequentaram sessões de simulação obtiveram melhorias nas habilidades de pensamento crítico ao longo do curso de enfermagem ${ }^{(29)}$.

Outro estudo da África do Sul revelou que os alunos percebem a simulação com pacientespadrão como uma ferramenta que preenche a lacuna entre a teoria e a prática podendo, a partir da simulação, aplicar os conhecimentos teóricos adquiridos. Além disso, o estudo identificou que os alunos identificam suas próprias potencialidades e fragilidades a partir da reflexão dos cenários vivenciados ${ }^{(30)}$.

No Brasil, uma pesquisa realizada em 2014, também mostrou que a simulação é estratégia geradora de satisfação entre os estudantes. $\mathrm{Na}$ mesma pesquisa, ainda foi constatado que a simulação proporciona uma vivência prévia da prática, que essa ferramenta permite pensar criticamente e refletir sobre a prática e contribui para relacionar a teoria à prática ${ }^{(31)}$.

Ao considerar a abrangência e utilização da simulação no ensino de enfermagem, bem como seu caráter de inserção e difusão recente na formação de profissionais de enfermagem, é importante conhecer os conceitos $e$ as características atribuídas ao fenômeno analisado.

Nessa perspectiva, faz-se necessário a clarificação de conceitos que podem contribuir para o ensino de enfermagem e, consequentemente, para a prática; uma vez que a identificação dos elementos de um conceito pode ser fundamental para a organização do conhecimento e, com isso, facilitar a sua aplicabilidade nos cenários de cuidado ${ }^{(32)}$.

Além disso, a análise contínua de conceitos derivados de outras disciplinas e que são incorporados pela a enfermagem é uma condição relevante para compreender o fenômeno, a sua aplicabilidade e particularidade na enfermagem e, consequentemente, estabelecer o seu campo específico ${ }^{(32)}$.

\section{Definição}

Com base na análise realizada, a simulação no ensino de enfermagem é identificada como: uma técnica de ensino que se utiliza de tecnologias para replicar cenários que simulam a prática, em ambiente controlado e realista, onde o estudante participa ativamente do processo de ensino e aprendizagem com a finalidade de praticar exaustivamente, aprender, refletir e avaliar produtos e processos.

\section{CONCLUSÃO}

A análise conceitual identificou o conceito simulação no ensino de enfermagem. A partir de uma revisão integrativa da literatura, identificouse que os atributos, antecedentes e consequentes foram os mais apontados na literatura. A partir dessa análise, foi possível identificar que o 
conceito estudado envolve: espaço que simula a prática; realismo; uso de manequins; feedback; repetição; técnica ou tecnologia; ambiente controlado; participação ativa do discente; praticar; aprender; avaliar; ferramenta tecnológica. A partir da análise desses atributos, foi criada uma definição.

Como limitação do estudo, considera-se a pequena quantidade de estudos internacionais. Além disso, buscaram-se estudos disponíveis em apenas três bases de dados. Recomendam-se novas pesquisas utilizando outros descritores e bases de dados. Apesar dessas limitações, a pesquisa trouxe contribuições importantes a partir da identificação dos atributos, antecedentes e consequentes.

Além disso, por ser derivado de outras áreas do conhecimento, o estudo poderá fornecer subsídios para a melhor compreensão, aplicação e uso da simulação enquanto técnica de ensino e ferramenta docente no contexto do ensino de Enfermagem. Assim, essa compreensão ampliada poderá contribuir na melhoria dos processos de ensino e aprendizagem, refletindo em melhor qualificação profissional. Além disso, a clarificação desse conceito poderá facilitar o desenvolvimento de instrumentos de pesquisas na área de investigação do fenômeno estudado para a organização do conhecimento nessa área de investigação, para a construção de declaração, afirmações e teorias de enfermagem.

\section{REFERÊNCIAS}

1. Jorge BM, Almeida RGS, Souza Junior VD. Tendências atuais na investigação em simulação. In: Martins JCA. A simulação no ensino de enfermagem. Coimbra: Unidade de Investigação em Ciências da Saúde: Enfermagem, Escola Superior de Enfermagem de Coimbra; 2014. p. 259-276.

2. Martins JCA, Mazzo A, Baptista RCN, Coutinho VRD, Godoy $S$, Mendes IAC, et al. A experiência clínica simulada no ensino de enfermagem: retrospectiva histórica. Acta Paul Enferm. 2012;25(4):619-25. DOI: 10.1590/S010321002012000400022

3. Sanino GEC. $O$ uso da simulação em enfermagem no Curso Técnico de Enfermagem. J Health Inform. 2012;4(nesp):148-51. Disponível em: $\quad$ http://www.jhi-sbis.saude.ws/ojsjhi/index.php/ihi-sbis/article/view/247

4. Walker L, Avant KC. Concept analysis. In: Walker L, Avant KC. Strategies for theory construction in nursing. $5^{\text {th }}$ ed. Prentice Hall; 2011. p. 35-50.

5. Whittemore R, Knafl K. Methodological issues in nursing research the integrative review: Updated methodology. J Adv Nursing. 2005 Dec;52(5):546-53. DOI: 10.1111/j.13652648.2005. 03621.x

6. Whittemore R. Combining evidence in nursing research: methods and implications. Nurs Res. 2005 Jan/Feb;54(1):56-62. Disponível em: https://www.ncbi.nlm.nih.gov/ pubmed/15695940

7. Bardin L. Análise de conteúdo. Lisboa: Edições 70; 1977.

8. Oliveira SNO, Prado ML, Kempfer SS. Utilização da simulação no ensino da enfermagem: Revisão integrativa. Rev Min Enferm. 2014 abr/jun;18(2):487-95. DOI: $10.5935 / 1415-$ 2762.20140036

9. Silveira RCP, Robazzi MLCC. Modelos e inovações em laboratórios de ensino em enfermagem. $R$ Enferm Cent $O$ Min. 2011 out/dez;1(4):592-602.

DOI:

10.19175/recom.v0i0.138

10. Santos MC, Leite MCL, Heck RM. A possibilidade de contribuição da acupuntura no ensino da simulação clínica em enfermagem. Rev Gaúcha Enferm. 2011 mar;32(1):185-8. DOI: 10.1590/S1983-14472011000100024

11. Marmol MT, Braga FTMM, Garbiri LM, Moreli L, Santos CB dos, Carvalho EC de. Curativo de cateter central em simulador: Efeito da presença do tutor ou da aprendizagem autoinstrucional. Rev Latino-Am Enfermagem 2012;20(6):11341141. DOI: $10.5935 / 1415-2762.20140036$

12. Alvarez AG, Dal Sasso GTM. Aplicação de objeto virtual de aprendizagem, para avaliação simulada de dor aguda, em estudantes de enfermagem. Rev Latino-Am Enfermagem 2011 mar/abr;19(2): 229-37. Disponível em: http://www.scielo.br/pdf/rlae/v19n2/pt 02

13. Salvador PT, Martins CCF, Alves KYA, Pereira MS, Tourinho FSV. Tecnologia no ensino de enfermagem. Ver Baiana Enfermagem 2015 jan/mar;29(1):33-41.

DOI: 10.18471/rbe.v29i1.9883

14. Pina-Jimenez I, Amador-Aguilar R. La enseñanza de laenfermeríacon simuladores, consideraciones teórico-pedagógicas para perfilar un modelo didáctico. Enferm Univ. 2015 jul/set;12(3):152-9.

DOI: 10.1016/j.reu.2015.04.007

15. Goes FSN, Fonseca LMM, Camargo RAA de, Hara CYN, Gobbi JD, Stabile AM. Elaboração de 
um ambiente digital de aprendizagem na educação profissionalizante em enfermagem. Cienc Enferm. 2015 abr;21(1):81-90. DOI: 10.4067/S0717-95532015000100008

16. Smithburger PL, et al. Advancing interprofessional education through the use of high fidelity human patient simulators. Pharm Pract. 2013 Apr;11(2):61-5. Disponível em: https://www.ncbi.nlm.nih.gov/pubmed/24155851 17. Teixeira INAO, Felix JVC. Simulação como estratégia de ensino em enfermagem: revisão de literatura. Interface 2011 out/dez;15(39):117384. DOI: $10.1590 / \mathrm{S} 1414-32832011005000032$

18. Costa RRO, Medeiros SM de, Martins JCA, Menezes RMP, Araújo MS de. O uso da simulação no contexto da educação e formação em saúde e enfermagem: uma reflexão acadêmica. Rev Espaço Saúde. 2015 jan/mar;16(1):59-65. DOI: 10.22421/1517-7130.2015v16n1p59

19. Santos MC, Leite MCL. A avaliação das aprendizagens na prática da simulação em Enfermagem como feedback de ensino. Rev Gaúcha Enferm 2010 set;31(3): 552-6. DOI: 10.1590/S1983-14472010000300020

20. Barreto DG, et al. Simulação realística como estratégia de ensino para o curso de graduação em enfermagem: Revisão integrativa. Rev Baiana Enferm. 2014 maio/ago28(2):208-14. Disponível em:

https://portalseer.ufba.br/index.php/enfermage $\mathrm{m}$ /article/viewFile/8476/8874

21. Almeida RGS, Mazzo A, Martins JCA, Pedersoli CE, Fumincelli L, Mendes IAC. Validação para a língua portuguesa da simulation design scale. Texto Contexto - Enferm. 2015;24(4):934-40. DOI: 10.1590/0104-0707201500004570014

22. Waterkemper R, Prado ML do. Estratégias de ensino-aprendizagem em cursos de graduação em Enfermagem. Av Enferm. 2011 Jul/Dic;29(2):234$46 . \quad$ Disponível em: http://www.bdigital.unal.edu.co/35461/1/35793142613-1-PB.pdf

23. Pazin FA, Scarpelini S. Simulação: Definição. Rev Medicina. 2007 abr/jun;40(2):162-6. Disponível em: http://revista.fmrp.usp.br/2007/vol40n2/2 simul acao definicao.pdf

24. Araújo ALLS, Quilici AP. O que é simulação e por que simular. In: Quilici $A P$, Abrão $K C$, Timerman S, Gutierrez F (Orgs). Simulação clínica do conceito a aplicabilidade. São Paulo: Editora Atheneu, 2012. p. 1-16.

25. Baptista RCN, Martins JCA, Pereira MFCR, Mazzo A. Students' satisfaction with simulated clinical experiences: Validation of an assessment scale. Rev. Latino-Am. Enfermagem. 2014 set/out;22(5):709-715. DOI: 10.1590/0104$\underline{1169.3295 .2471}$

26. Arthur C, Levett-Jones T, Kable A. Quality indicators for the design and implementation of simulation experiences: A Delphi study. Nurse Educ Today 2013 Nov;33(11):1357-61. DOI: 10.1016/i.nedt.2012.07.012.

27. Berragan L. Simulation: An effective pedagogical approach for nursing? Nurse Educ Today 2011 Oct;31(7):660-3. DOI: 10.1016/i.nedt.2011.01.019

28. Lima MAC, Cassiani SHB. Pensamento crítico: Um enfoque na educação de enfermagem. Rev Latino-Am Enfermagem 2000 jan;8(1):23-30. DOI: 10.1590/S0104-11692000000100004

29. Kelly SH. Evaluation methods used in simulation: a survey of faculty and student perceptions in an undergraduate nursing programa [tese]. Pittsburgh (PA): School of Education the University of Pittsburgh; 2014.

30. Botma Y. Nursing student's perceptions on how immersive simulation promotes theorypractice integration. IJANS. 2014;1:1-5. DOI: 10.1016/j.ijans.2014.04.001

31. Costa RRO. A simulação realística como estratégia de ensino-aprendizagem em enfermagem [dissertação]. Natal (RN): Universidade Federal do Rio Grande do Norte; 2014.

32. Fernandes MGM, Nóbrega MML, Garcia TR, Macêdo-Costa KNF. Análise conceitual: Considerações metodológicas. Rev Bras Enferm. 2011 jan;64(6):1150-6. DOI: 10.1590/141381232017221.13522015

Nota: O presente trabalho é parte da construção do referencial teórico do projeto de pesquisa intitulado "Eficácia da simulação realística no ensino de imunização de adultos no contexto da graduação em enfermagem", em desenvolvimento do Programa de Pós-Graduação em Enfermagem da Universidade Federal do Rio Grande do Norte-UFRN.

Recebido em: $20 / 04 / 2017$

Aprovado em: 11/05/2018

Endereço de correspondência:

Raphael Raniere de Oliveira Costa

Rua Pedras Grandes, 1982 - Potengí

CEP: 59.110-010 - Natal/RN - Brasil

E- mail: raphaelraniere@hotmail.com 